\title{
Regulation of Keratocyte Phenotype and Cell Behavior by Substrate Stiffness
}

\author{
Jialin Chen,* Ludvig J. Backman, Wei Zhang, Chen Ling, and Patrik Danielson* \\ Cite This: ACS Biomater. Sci. Eng. 2020, 6, 5162-5171 \\ Read Online
}

ABSTRACT: Corneal tissue engineering is an alternative way to solve the problem of lack of corneal donor tissue in corneal transplantation. Keratocytes with a normal phenotype and function in tissue-engineered cornea would be critical for corneal regeneration. Although the role of extracellular/substrate material stiffness is well-known for the regulation of the cell phenotype and cell behavior in many different cell types, its effects in keratocyte culture have not yet been thoroughly studied. This project studied the effect of substrate stiffness on the keratocyte phenotype marker expression and typical cell behavior (cell adhesion, proliferation, and migration), and the possible mechanisms involved. Human primary keratocytes were cultured on tissue culture plastic (TCP,

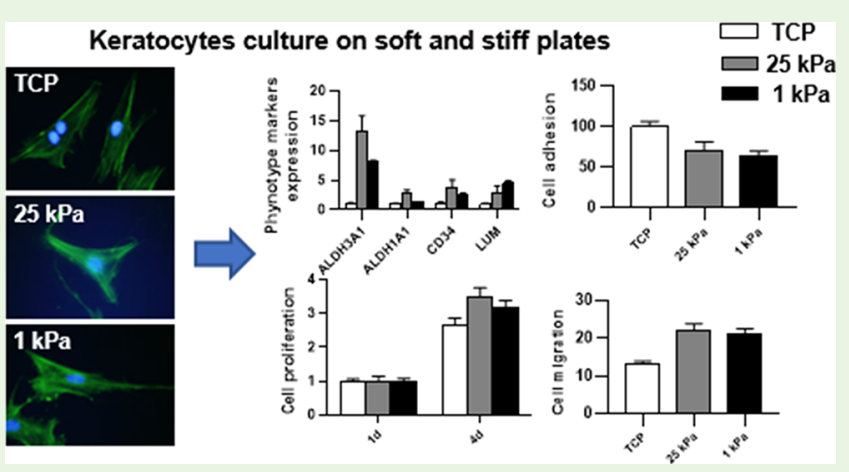
$\sim 10^{6} \mathrm{kPa}$ ) or on plates with the stiffness equivalent of physiological human corneal stroma $(25 \mathrm{kPa})$ or vitreous body $(1 \mathrm{kPa})$. The expression of keratocyte phenotype markers, cell adhesion, proliferation, and migration were compared. The results showed that the stiffness of the substrate material regulates the phenotype marker expression and cell behavior of cultured keratocytes. Physiological corneal stiffness $(25 \mathrm{kPa})$ superiorly preserved the cell phenotype when compared to the TCP and $1 \mathrm{kPa}$ group. Keratocytes had a larger cell area when cultured on $25 \mathrm{kPa}$ plates as compared to on TCP. Treatment of cells with NSC 23766 (Rac1 inhibitor) mimicked the response in the cell phenotype and behavior seen in the transition from soft materials to stiff materials, including the cytoskeletal structure, expression of keratocyte phenotype markers, and cell behavior. In conclusion, this study shows that substrate stiffness regulates the cell phenotype marker expression and cell behavior of keratocytes by Rac1-mediated cytoskeletal reorganization. This knowledge contributes to the development of corneal tissue engineering.

KEYWORDS: keratocytes, stiffness, phenotype, cell behavior, cytoskeletal reorganization, Rac1

\section{INTRODUCTION}

Corneal blindness due to trachoma, onchocerciasis, and vitamin A deficiency is common worldwide. ${ }^{1}$ Corneal transplantation is the main method for visual rehabilitation when there are profound scars in the cornea. However, the shortage of corneal donor tissue limits its application, especially in developing countries. In China, around 5000 corneal transplantations are performed annually. ${ }^{2}$ However, the number of patients with corneal blindness is approximately 4-5 million. ${ }^{3}$ To solve the problem of long waiting lists for corneal transplantation, artificial corneal tissue is being developed by scientists, using the concept of tissue engineering. The stroma is the main part of the cornea (90\% of the tissue), which consists of collagen fibers and quiescent keratocytes. Upon injury, the quiescent keratocytes are stimulated to become fibroblasts and myofibroblasts to facilitate wound healing. ${ }^{4}$ This kind of self-repair is meaningful in terms of evolution, which protects the injured corneal tissue from further damage. Nevertheless, the functional recovery of injured cornea is seldom achieved because of the phenotype drift of keratocytes. ${ }^{5}$ During the transformation of keratocytes to fibroblasts and myofibroblasts, the expression of keratocyte phenotype markers is reduced or diminished, such as aldehyde dehydrogenase 3A1 (ALDH3A1), CD34, and keratocan (KERA). ${ }^{4}$ Transplantation of tissue-engineered cornea can rapidly fill the injured area. However, the keratocyte phenotype in the transplant determines the quality of corneal repair. Therefore, preservation of the keratocyte phenotype in tissueengineered cornea is critical for the quality and function of the transplant.

Microenvironment regulates the cell phenotype and cell fate. ${ }^{6,7}$ The fact that the cornea is a structure subjected to pressure results in corneal cells receiving a dome-shaped

Received: April 8, 2020

Accepted: July 30, 2020

Published: July 30, 2020 
mechanical strain. Our previous study showed that domeshaped mechanical strain enhances the expression of keratocyte markers, including lumican (LUM), KERA, and collagen type I (COL I) and type V (COL V). ${ }^{8}$ Substrate stiffness, a microcosmic mechanical factor caused by the extracellular matrix or contacted materials, is therefore assumed to be of importance for the phenotype preservation of corneal cells. When human corneal epithelial cells are cultured on substrates with different stiffness, obvious differences in the cell phenotype and behavior are seen, such as differences in the biomarker expression, cell apoptosis, cytoskeleton structure, and cell migration.,10 The physiological stiffness of the human corneal stroma is $24-39 \mathrm{kPa},{ }^{11}$ which is significantly lower than the material stiffness of commercial tissue culture plastic (TCP, around $10^{6} \mathrm{kPa}$ ). Dreier et al. cultured human and rabbit corneal fibroblasts in transforming growth factor $\beta 1$ (TGF- $\beta 1$ )-supplemented medium. They found that cells expressed significantly less $\alpha$ smooth muscle actin ( $\alpha$-SMA, a key marker of myofibroblast transformation) when they were cultured on more compliant (soft) substrates $(4-71 \mathrm{kPa})$, as compared to those cultured on TCP. ${ }^{12}$ Lakshman and Petroll studied the mechanical phenotype of cultured rabbit keratocytes. They found that $\operatorname{TGF} \beta$ stimulates the transformation of keratocytes to myofibroblasts, which could be enhanced by increased substrate stiffness. ${ }^{13}$ Therefore, stiff substrates, such as TCP, are nonoptimal for in vitro corneal study. Nevertheless, the effect of physiological stiffness on the preservation of the keratocyte phenotype (expression of phenotype markers) and on the typical cell behavior (cell adhesion, proliferation, and migration) has not yet been investigated and needs to be studied thoroughly.

Cytoskeletal remodeling is important for the cell phenotype and cell behavior. ${ }^{14,15}$ The cytoskeleton is sensitive to mechanical stimuli, including stiffness, and the Rho-family of small GTPases, which regulates cytoskeletal remodeling, responds to the signals of mechanical stimuli by inducing the appropriate remodeling. ${ }^{16-18}$ Ras-related C3 Botulinum Toxin Substrate 1 (Rac1) and Rho-kinase (ROCK) are central members of the Rho-family. In a previous study, Petroll and collaborators found that Racl and ROCK were involved in the transformation of cultured keratocytes toward the fibroblast and myofibroblast phenotype. ${ }^{5}$ However, their role in stiffnessinduced alteration of the keratocyte phenotype marker expression and cell behavior is not yet well-known.

The hypothesis of this study is that substrate stiffness regulates the keratocyte phenotype marker expression and cell behavior by cytoskeletal reorganization. To test this hypothesis, human primary keratocytes were cultured on TCP $\left(\sim 10^{6}\right.$ $\mathrm{kPa})$, or on plates with the stiffness equivalent of physiological human corneal stroma $(25 \mathrm{kPa})$ and vitreous body $(1 \mathrm{kPa})$. The expression of keratocyte phenotype markers was compared, as well as various cell behaviors, such as cell adhesion, proliferation, and migration. The role of cytoskeletal remodeling and the involvement of Rac1/ROCK were also studied by inhibitor treatment.

\section{MATERIALS AND METHODS}

2.1. Cell Culture. The collection of human material for study purpose was vetted by the Regional Ethical Review Board in Umeå, which determined it to be exempt from the requirement for approval $(2010-373-31 \mathrm{M})$. As previously described, ${ }^{8}$ primary human limbal keratocytes were isolated and cultured in DMEM/F-12 (Life technologies, Grand Island, New York, USA, \#21331-046) supplemented with 2\% FBS (Life technologies, \#16000) and 1\% penicillin-streptomycin (Life Technologies, \#15410). Only cells before passage three were used in the experiments of this study.

For TCP versus Collagen I Coated Plates (CCP), human keratocytes $\left(3.1 \times 10^{4}\right.$ cells $\left./ \mathrm{cm}^{2}\right)$ were seeded on TCP (Sarstedt, Helsingborg, Sweden, \#83.3920.005) and CCP (Life technologies, A11428-01), and cultured for 3 and 7 days. At the designated time points, $\mathrm{mRNA}$, and protein were extracted for quantitative polymerase chain reaction (qPCR) and Western blot analysis.

For TCP versus $25 \mathrm{kPa}$ versus $1 \mathrm{kPa}$, Softwell plates and Softslip coverslips (Cell Guidance Systems, Cambridge, UK) were used. Softwell plates and Softslip coverslips are commercial hydrogel-coated products with controlled stiffness (e.g., 25 and $1 \mathrm{kPa}$ ). The variation in stiffness is achieved by modifying the crosslink of polyacrylamide and bisacrylamide. As the manufactory describes, Collagen I was chemically conjugated to polyacrylamide gels to enable cell attachment and growth, and the stiffness of these products were quantified by their elastic modulus (E). In this study: (1) Keratocytes $\left(3.1 \times 10^{4}\right.$ cells $/ \mathrm{cm}^{2}$ ) were seeded in TCP 6-well plate, 25 and $1 \mathrm{kPa}$ Softwell 6well plates (SW6-COL-25 EA and SW6-COL-1 EA). After 3 days in culture, cells were collected for qPCR assay or transferred to transwell $\left(1 \times 10^{5} /\right.$ well, Cell Biolabs, San Diego, CA, CBA-100) for cell migration assay after another 24 hours. $^{19}(2)$ Keratocytes $\left(6.2 \times 10^{3}\right.$ cells $\left./ \mathrm{cm}^{2}\right)$ were seeded in the TCP 96-well plate, 25 and $1 \mathrm{kPa}$ Softwell 96-well plate (SW96-COL-25 EA and SW96-COL-1 EA). After 1 and 4 days of culture, the CellTiter $96 \mathrm{AQ}_{\text {ueous }}$ One Solution Cell Proliferation Assay (Promega, Fitchburg, WI, G3581) was used to determine cell adhesion ${ }^{20}$ and proliferation. (3) Keratocytes (7000 cells $/ \mathrm{cm}^{2}$ ) were seeded in 8 well chamber slides (Corning, NY, USA \#354118), and Softslip 24 coverslips with stiffness of $25 \mathrm{kPa}$ and 1 $\mathrm{kPa}$ (SS24-COL-25 EA and SS24-COL-1 EA). After $24 \mathrm{~h}$ of culture, F-actin staining was performed. After 3 days of culture, immunofluorescence staining of Ki67 (Table S1) was performed.

For control (Ctrl) versus cytochalasin D (Cyto D) or NSC 23766 or Y-27632, $3.1 \times 10^{4}$ cells $/ \mathrm{cm}^{2}$ keratocytes were seeded and left to adhere for 1 day before treated with $50 \mu \mathrm{M}$ Cyto D (Tocris, Bristol, $\mathrm{UK}$ \#1233), or $50 \mu \mathrm{M}$ NSC 23766 (Tocris, \#2161), or $10 \mu \mathrm{M} \mathrm{Y}$ 27632 (Sigma-Aldrich, St. Louis, Missouri, USA, Y0503), or untreated. After 3 days of culture, cells were collected for the qPCR assay. Meanwhile, in the group with or without Cyto D treatment, pictures of the cell morphology were taken under a light microscope. To compare the groups with and without NSC 23766 treatment, Factin staining was performed at day 3 (initial cell seeding density was 3000 cells $/ \mathrm{cm}^{2}$ ), cell adhesion and proliferation were evaluated at day 1 and day 4 (initial cell seeding density was $6.2 \times 10^{3}$ cells $/ \mathrm{cm}^{2}$ ). After 3 days of treatment, cells were also transferred to transwell $(1 \times$ $10^{5} /$ well) for the cell migration assay after another $24 \mathrm{~h}$.

2.2. qPCR Assay. RNA extraction and cDNA reverse transcription were performed as previously described. ${ }^{21}$ TaqMan Gene Expression Assay (Applied Biosystems, Carlsbad, USA) and SYBR Green reagents (Applied Biosystems) were used for qPCR experiments. All used probes and primers are summarized in Tables S2 and S3. Representative results of cells from at least two individuals are displayed as target gene expression normalized to housekeeping gene.

2.3. Western Blot Analysis. Protein extraction and Western blot experiments were performed as previously described. ${ }^{22}$ Cells from three replicate wells were pooled together as one sample. The densitometry of bands was quantified by ImageJ analysis software $(\mathrm{NIH})$. All antibodies used for Western blot are summarized in Table S1.

2.4. Cell Adhesion and Proliferation Assay. At designated time points, cells were incubated with CellTiter $96 \mathrm{AQ}_{\text {ueous }}$ One Solution Reagent for $1 \mathrm{~h}$ at $37{ }^{\circ} \mathrm{C}$ in a $5 \% \mathrm{CO}_{2}$ incubator. The absorbance of the culture medium was subsequently measured at $490 \mathrm{~nm}$.

2.5. Immunofluorescence Staining. Immunofluorescence staining of Ki67 was carried out as previously described. ${ }^{23}$ DAPI was used to reveal the nuclei of the cells.

2.6. Cell Migration Assay. Before the cell migration assay, cells were pretreated as described in detail above (Section 2.1). Cell 
A

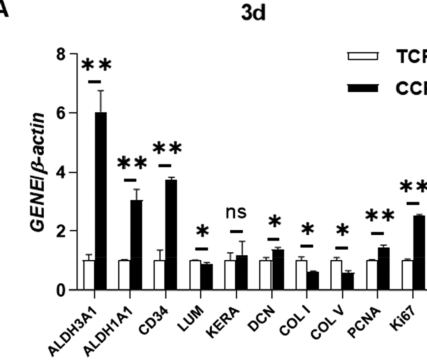

C

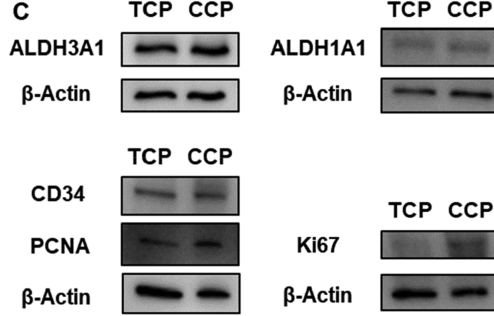

E

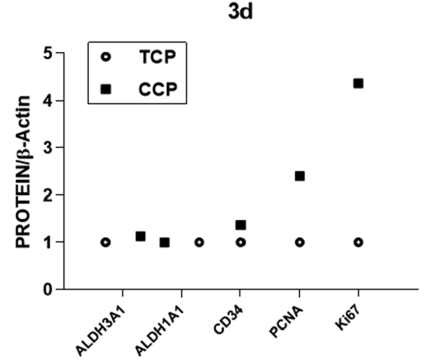

B
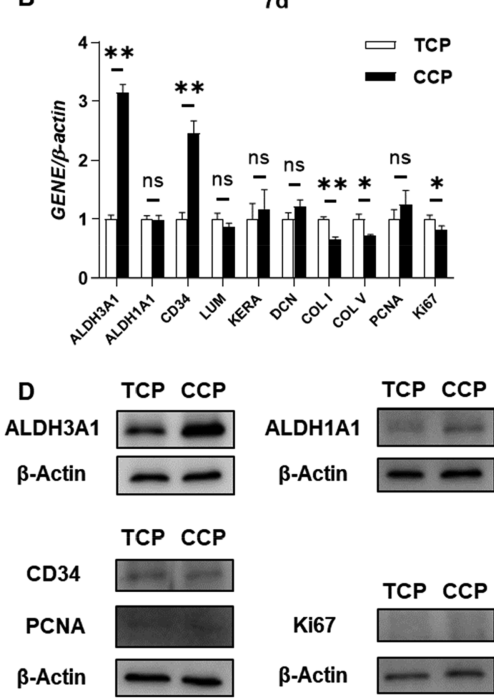

F

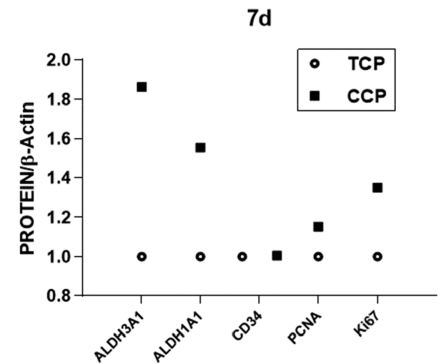

Figure 1. Collagen-coated plate (CCP) promotes the expression of keratocyte phenotype markers and proliferation markers. Human keratocytes were cultured on TCP and CCP for 3 (3d) and 7 days (7d). Gene expression between the two groups was compared by qPCR after 3 (A) and 7 days (B). Levels in the TCP group were set as $1 . *_{p}<0.05 . * *_{p}<0.001$. ns no significant difference $(p \geq 0.05)$. Protein expression between two groups was compared by Western blot after 3 (C) and 7 days (D). Densitometry was quantified, and the ratio of protein/ $\beta$-actin was calculated $(\mathrm{E}, \mathrm{F})$. Levels in the TCP group were set as 1.

migration assay was performed according to the manufacturer's protocol. Briefly, cell suspension solution was added to the inside of each insert and incubated for $24 \mathrm{~h}$. The media inside the insert was then removed and nonmigratory cells were also removed by cottontipped swabs. Subsequently, the insert with migratory cells was stained and detached. The solution was measured at $480 / 520 \mathrm{~nm}$.

2.7. Cytoskeletal Structure Analysis. Cytoskeletal structure was revealed by F-actin staining with BODIPY FL Phallacidin (Invitrogen, Carlsbad, California, USA, B607) for $30 \mathrm{~min}$. DAPI was used to show the nuclei of the cells. The area and aspect ratio (length/width) of cells were measured and calculated by Image $\mathrm{J} \cdot{ }^{23}$ At least 35 representative cells were measured for statistical analysis.

2.8. Statistical Analysis. Data are shown as mean \pm SD unless otherwise specially declared. Student's $t$-test was used for two-group comparison. One-way ANOVA, followed by pair-wise comparisons with Bonferroni post-hoc correction between groups of interest, was used in experiments with more than two groups. Experiments were performed in triplicate and were repeated successfully. In total, human keratocytes derived from 19 patient samples were used in this study. Representative results are shown. For all comparisons, $p<0.05$ was considered statistically significant.

\section{RESULTS}

3.1. Collagen-Coated Plate Promotes the Expression of Keratocyte Phenotype Markers and Proliferation Markers. Human keratocytes were cultured on TCP and CCP for 3 and 7 days. Expression of typical keratocyte phenotype markers was compared, including $A L D H 3 A 1, A L D H 1 A 1$, CD34, LUM, and KERA. ALDH is referred to as corneal crystalline and contributes to corneal transparency. CD34 functions as an adhesion molecule and is another typical phenotype marker of keratocytes. LUM and KERA are both major proteoglycans that are present in the corneal stroma and expressed by keratocytes. The expression of genes for keratocyte phenotype markers was significantly upregulated in the CCP group at day 3 (Figure $1 \mathrm{~A} A L D H 3 A 1,6.04$-fold, $p$ $<0.001$; ALDH1A1, 3.05-fold, $p<0.001$; CD34, 3.73-fold, $p<$ 0.001 ) and day 7 (Figure 1B ALDH3A1, 3.15-fold, $p<0.001$; CD34, 2.46-fold, $p<0.001$ ), as compared to that in the TCP group. Cells in the CCP group expressed lower collagen genes (COL $I$ and $C O L V$ ), but higher DCN, than cells in the TCP group (Figure 1A,B). Furthermore, the expression of genes for proliferation markers was significantly upregulated in the CCP group at day 3 (Figure 1A PCNA, 1.45-fold, $p<0.001$; Ki67, 2.53 -fold, $p<0.001)$, as compared to that in the TCP group. Western blot results showed that protein expression of proliferation markers was increased in the CCP group at day 3 , as compared to that in the TCP group (Figure 1C,E PCNA, 2.40-fold; Ki67, 4.36-fold). At day 7, the protein expression of proliferation markers between the two groups was not as obvious as in day 3 (Figure $1 \mathrm{D}, \mathrm{F}$ as compared to $1 \mathrm{C}, \mathrm{E}$ ). However, the protein expression of keratocyte phenotype markers ALDH3A1 and ALDH1A1 was much higher in the CCP group than in the TCP group (1.86-fold and 1.55-fold, respectively) at day 7 (Figure 1D,F). Expression of $\alpha$-SMA was 
A

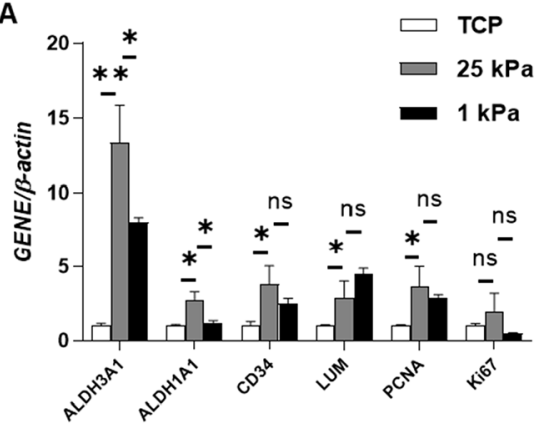

B $\square$ TCP

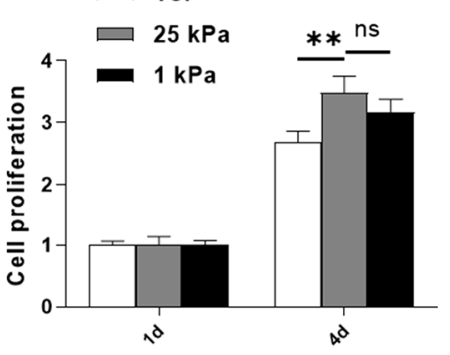

C
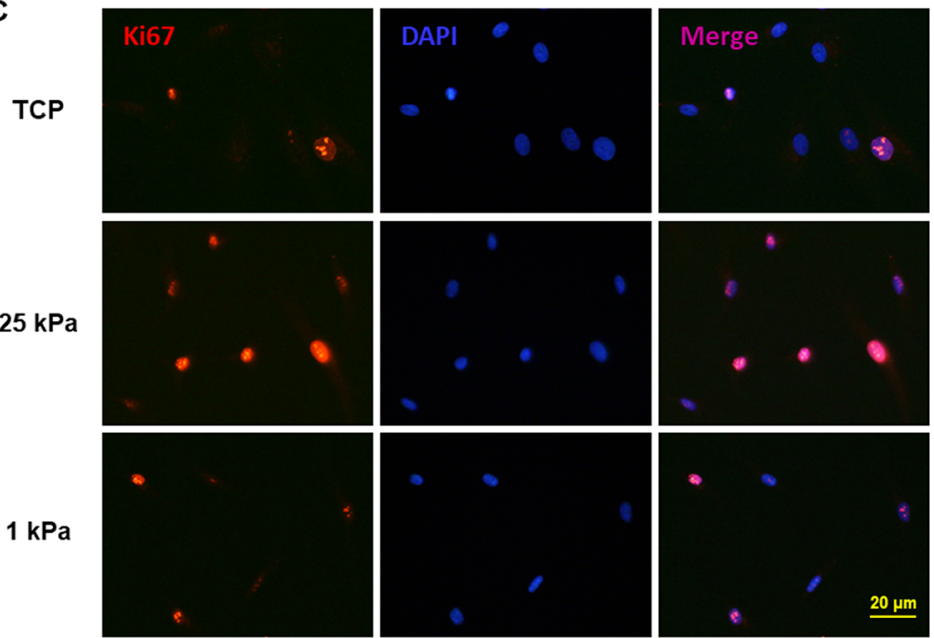

Figure 2. Physiological stiffness of corneal stroma is beneficial for keratocyte phenotype markers expression and proliferation. Human keratocytes were cultured on TCP $\left(\sim 10^{6} \mathrm{kPa}\right), 25 \mathrm{kPa}$ plates (stiffness of physiological human corneal stroma), and $1 \mathrm{kPa}$ plates (stiffness of physiological human vitreous body). (A) Gene expression was evaluated at day 3 by qPCR. Levels in the TCP group were set as 1 . The expression was compared for $25 \mathrm{kPa}$ vs TCP and $25 \mathrm{kPa}$ vs $1 \mathrm{kPa}$. (B) The proliferation rate was compared. Levels at 1 day (1d) were set as 1 . The comparison was carried out for $25 \mathrm{kPa}$ vs TCP and $25 \mathrm{kPa}$ vs $1 \mathrm{kPa}$. (C) Immunofluorescence staining was performed to analyze the expression of Ki67 between groups after 3 days of culture. The right column is the merged picture of the left column and middle column. $* p<0.05$. $* * p<0.001$. ns no significant difference $(p \geq 0.05)$.

also evaluated. However, only a very low expression was found (data not shown).

Two possible factors could explain the difference between TCP and CCP. One is substrate stiffness, the other is the collagen itself as an ECM component. The effect of the ECM components (COL I and COL V) on the expression of keratocyte phenotype markers was evaluated, and no obvious differences were found between the ECM treated groups as compared to the control group (Figure S1). Therefore, the effect of substrate stiffness on keratocytes was further evaluated.

3.2. Physiological Stiffness of Corneal Stroma is Beneficial for Keratocyte Phenotype Marker Expression and Proliferation. To evaluate the effect of substrate stiffness on the keratocyte phenotype marker expression and cell behavior, human keratocytes were cultured on plates with different stiffness. The different stiffness plates used were TCP $\left(\sim 10^{6} \mathrm{kPa}\right.$ ), $25 \mathrm{kPa}$ plates (stiffness of physiological human corneal stroma), and $1 \mathrm{kPa}$ plates (stiffness of physiological human vitreous body). After 3 days of culture, the $25 \mathrm{kPa}$ group had significantly higher expression of ALDH3A1 ( $p<$ 0.001 vs TCP; $p<0.05$ vs $1 \mathrm{kPa}$ ) and $A L D H 1 A 1$ ( $p<0.05$ vs TCP and $1 \mathrm{kPa}$ ) as compared to the other two groups (Figure 2A). The enhanced expression for $A L D H 3 A 1$ and $A L D H 1 A 1$ was found to be 13.32-fold and 2.75-fold, respectively, in physiological corneal stiffness (25 kPa group), as compared to
TCP. Physiological vitreous body stiffness (1 $\mathrm{kPa}$ group) also promoted the expression of $A L D H 3 A 1$ (8.03-fold of TCP) and ALDH1A1 (1.21-fold of TCP), but to a lesser extent than in the $25 \mathrm{kPa}$ group. The substrate with physiological corneal stiffness $(25 \mathrm{kPa})$ also promoted the gene expression of CD34 $(p<0.05)$, LUM $(p<0.05)$ and proliferation marker PCNA $(p$ $<0.05)$, as compared to TCP. The cell proliferation rate was evaluated (Figure 2B). Although no significant difference was found between the $25 \mathrm{kPa}$ group and the $1 \mathrm{kPa}$ group ( $p=$ $0.055)$, it was noticed that cells in the $25 \mathrm{kPa}$ group had significantly higher proliferation ( $30 \%$ faster) than the TCP group at day 4. Similar conclusions could be drawn from immunofluorescence of proliferation marker Ki67 on the protein level (Figure 2C).

3.3. Substrate Stiffness Regulates Cell Adhesion and Migration of Cultured Keratocytes. The effect of substrate stiffness on cell adhesion and migration was evaluated (Figure $3)$. As compared to the TCP group, the cell adhesion ratio of the $25 \mathrm{kPa}$ group was significantly lower $(71.34 \%, p<0.001)$ (Figure 3A). However, there was no significant difference between the $25 \mathrm{kPa}$ group and the $1 \mathrm{kPa}$ group $(p \geq 0.05)$. Integrins play a role in cell adhesion regulated by mechanics. ${ }^{24}$ Four well-known integrins were evaluated in this study (Figure 3B). The expression of ITGA1 $(p<0.001)$, ITGA5 $(p<0.05)$, and ITGB1 $(p<0.05)$ was downregulated in the $25 \mathrm{kPa}$ group, as compared to the TCP group. No significant difference of 
A

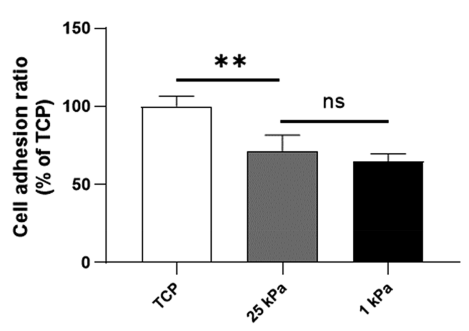

C

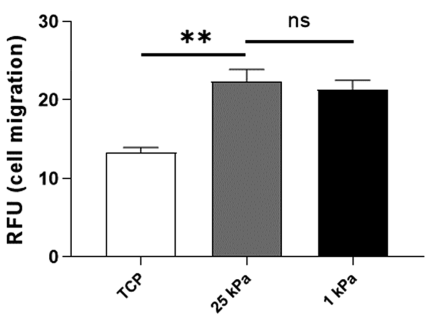

B
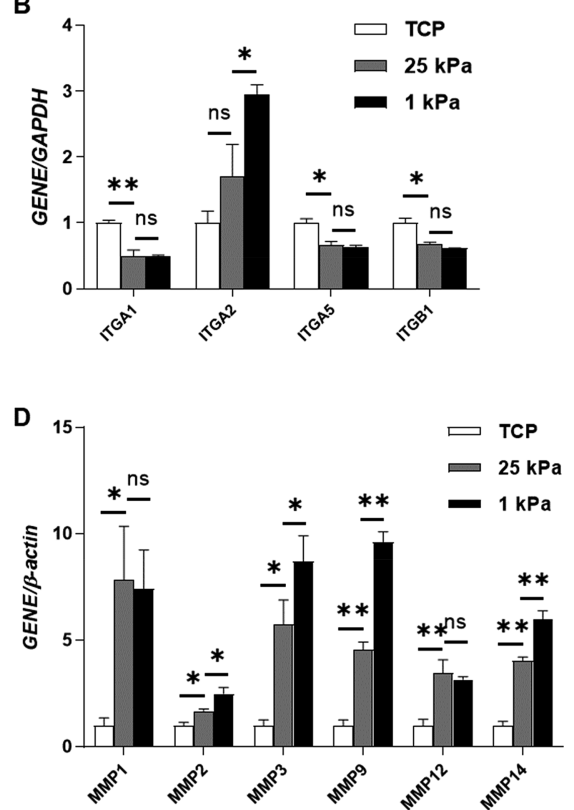

Figure 3. Substrate stiffness regulates cell adhesion and migration of cultured keratocytes. (A) Human keratocytes were seeded on TCP $\left(\sim 10^{6}\right.$ $\mathrm{kPa}$ ), $25 \mathrm{kPa}$ plates, and $1 \mathrm{kPa}$ plates. After $24 \mathrm{~h}$, the cell adhesion ability was evaluated. The level in the TCP group was set as $100 \%$. (B) Gene expression of integrins after 3 days of culture. The level of expression in the TCP group was set as 1. (C) Keratocytes were cultured on plates with different stiffness for 3 days. Cell migration ability was measured by transferring cells to a transwell insert and analyzed after $24 \mathrm{~h}$. (D) Gene expression of MMPs after 3 days of culture. The level of expression in the TCP group was set as 1 . All comparisons were performed between $25 \mathrm{kPa}$ and TCP, and between 25 and $1 \mathrm{kPa} . *_{p}<0.05$. $* * p 0.001$. ns no significant difference $(p \geq 0.05)$.

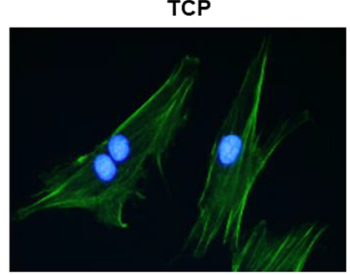

B

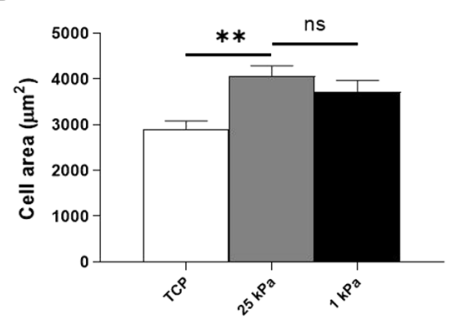

D

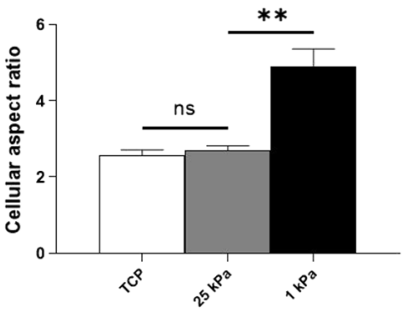

$25 \mathrm{kPa}$
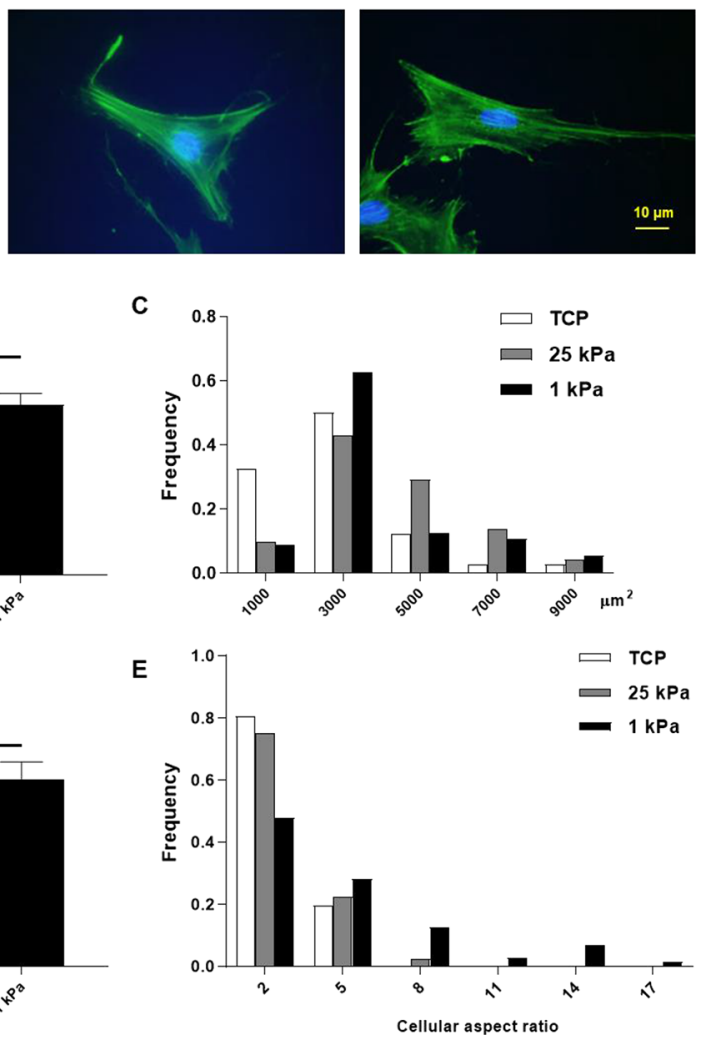

Figure 4. Substrate stiffness regulates cytoskeletal organization. Human keratocytes were cultured for $24 \mathrm{~h}$ on TCP $\left(\sim 10^{6} \mathrm{kPa}\right), 25 \mathrm{kPa}$ plates, and $1 \mathrm{kPa}$ plates. (A) F-actin staining (green) was performed to show the cytoskeletal structure of cultured cells. DAPI (blue) was used to reveal the nuclei of the cells. Cell area was quantified by Image J and shown as mean cell area (B) and cell area distribution (C). Cellular aspect ratio (length/ width) was also quantified by Image $J$ and shown as mean aspect ratio (D) and aspect ratio distribution (E). Results are shown as mean \pm SEM. All comparisons were performed between $25 \mathrm{kPa}$ and TCP and between 25 and $1 \mathrm{kPa}$. ** $p<0.001$. ns no significant difference $(p \geq 0.05)$. 
A

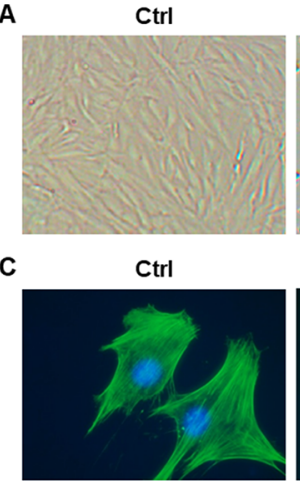

D

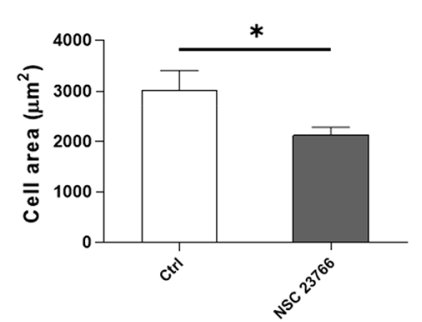

$\mathbf{F}$

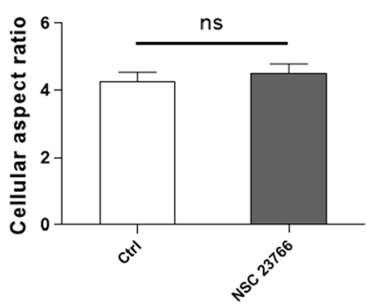

B

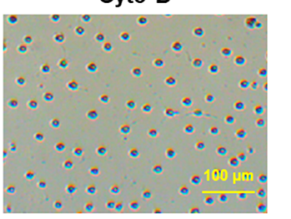

NSC 23766

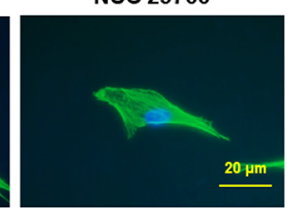

$\square \mathrm{Ctrl}$

NSC 23766

- Y-27632

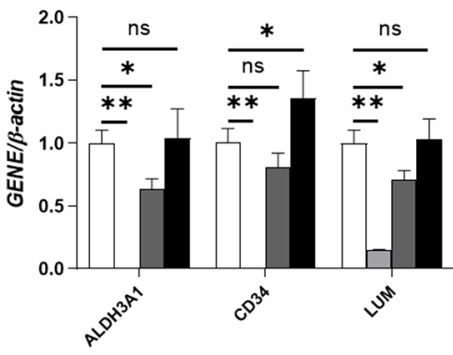

E $0.87 \quad \square$ Ctrl

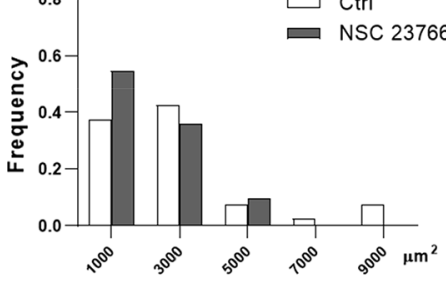

G $\quad 0.8-7$ Ctrl

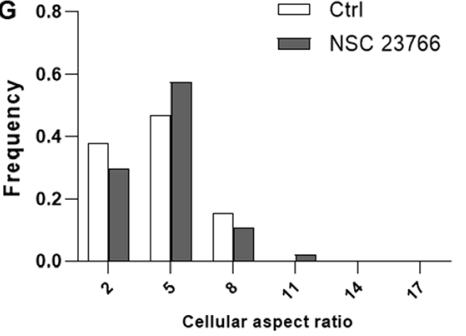

Figure 5. Cytoskeletal organization and keratocyte phenotype marker expression were regulated by NSC 23766 (Rac1 inhibitor). (A) Cultured human keratocytes were treated with or without $50 \mu \mathrm{M}$ Cyto D for 3 days. (B) Cells were treated with $50 \mu \mathrm{M}$ Cyto D, or $50 \mu \mathrm{M}$ NSC 23766 , or 10 $\mu \mathrm{M}$ Y-27632, or untreated (control group; Ctrl). After 3 days of treatment, samples were collected for the qPCR assay. Levels in the Ctrl group were set as 1 . All other groups were compared to the Ctrl group. (C) F-actin staining (green) was performed at day 3 to show the cytoskeletal structure of cultured cells. DAPI (blue) was used to reveal the nuclei of the cells. Cell area was quantified by Image J and shown as mean cell area (D) and cell area distribution (E). Cellular aspect ratio (length/width) was also quantified by Image J and shown as mean aspect ratio (F) and aspect ratio distribution $(\mathrm{G})$. Results are shown as mean \pm SEM. Ctrl, control group. $*_{p}<0.05$. $* *_{p}<0.001$. ns no significant difference $(p \geq$ $0.05)$.

ITGA2 was found between the $25 \mathrm{kPa}$ and the TCP group. As for cell migration ability, an increase of $67.52 \%(p<0.001)$ was shown in the $25 \mathrm{kPa}$ group as compared to the TCP group (Figure 3C). No significant difference was noticed between the $25 \mathrm{kPa}$ and the $1 \mathrm{kPa}$ group $(p \geq 0.05)$. Matrix metalloproteinases (MMPs) are generally thought to mediate cell migration. ${ }^{21,25}$ Six MMPs were evaluated in this study (Figure 3D). Interestingly, the expression of all MMPs detected was upregulated $(p<0.05$ or $p<0.001)$ in the $25 \mathrm{kPa}$ group as compared to that in the TCP group, which is consistent with their migration ability. Cells in the $1 \mathrm{kPa}$ group showed significantly higher levels of expression of MMP2 $(p<0.05)$, MMP3 ( $p<0.05)$, MMP9 $(p<0.001)$, and MMP14 $(p<$ 0.001 ), as compared to in the $25 \mathrm{kPa}$ group.

3.4. Substrate Stiffness Regulates Cytoskeletal Organization. The cytoskeletal structure of a cell mediates the mechanical signal transmission from the substrate stiffness to intracellular pathways, and is thereby involved in the altering of the cell's phenotype and cell behavior caused by the substrate stiffness. ${ }^{26}$ Human keratocytes were cultured for $24 \mathrm{~h}$ on TCP $\left(\sim 10^{6} \mathrm{kPa}\right), 25 \mathrm{kPa}$ plates, and $1 \mathrm{kPa}$ plates. F-actin staining was performed to show the cytoskeletal structure of the cultured cells (Figure 4A). Cell area and the cellular aspect ratio were quantified (Figure $4 \mathrm{~B}-\mathrm{E}$ ). It was found that cells cultured on $25 \mathrm{kPa}$ plates possessed larger cell area (1.40-fold, $p<0.001$ ), but had similar cellular aspect ratio (1.04-fold, $p \geq$ $0.05)$, as compared to cells cultured on TCP plates. No significant difference of quantified cell area was found between the $25 \mathrm{kPa}$ group and the $1 \mathrm{kPa}$ group $(p \geq 0.05)$. However, the cellular aspect ratio was significantly higher in the $1 \mathrm{kPa}$ group (1.82-fold, $p<0.001$ ), as compared to in the $25 \mathrm{kPa}$ group.

3.5. NSC 23766 (Rac1 Inhibitor) Regulates Cytoskeletal Organization and Keratocyte Phenotype Marker Expression. Cytochalasin D (Cyto D) is a small molecule that disrupts the actin filaments in cells. ${ }^{27}$ To further understand the relationship between the cytoskeletal structure and keratocyte phenotype marker expression, Cyto D was used to treat cultured keratocytes. Cells became round after Cyto D treatment, an obvious difference as compared to the control group in the cell shape (Figure 5A). The expression of keratocyte phenotype markers significantly decreased after Cyto D treatment (ALDH3A1, 0.00\%, $p<0.001$; CD34, $1.00 \%, p<0.001 ;$ LUM, 14.67\%, $p<0.001$ ) (Figure 5B). The 
A

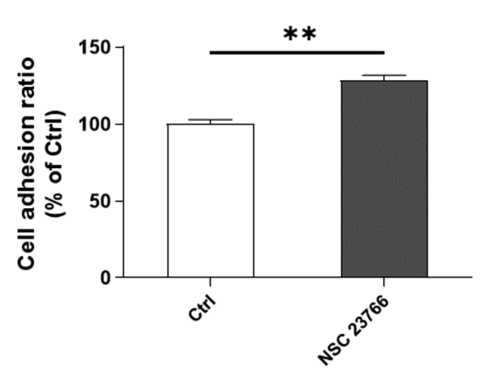

C

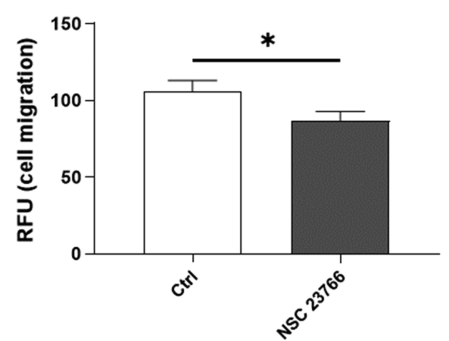

B
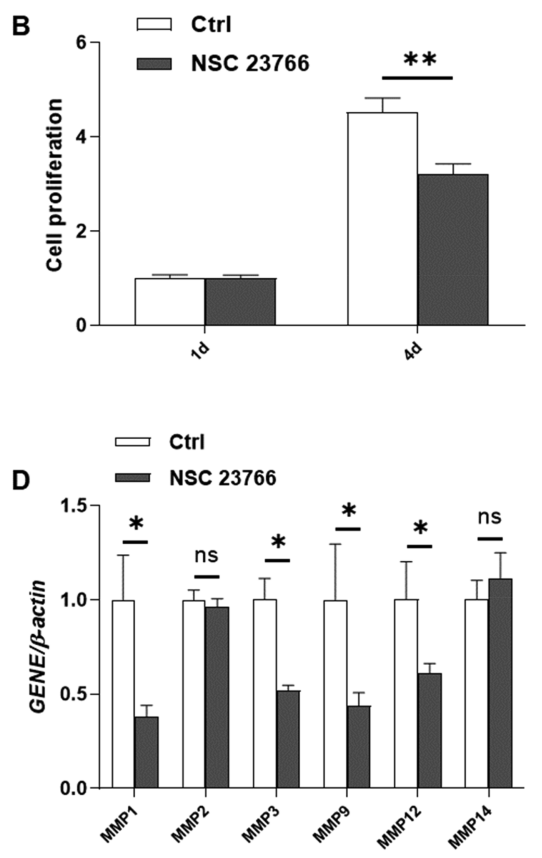

Figure 6. NSC 23766 regulates cell adhesion, proliferation, and migration of cultured keratocytes. Cells were treated with $50 \mu \mathrm{M}$ NSC 23766 or left untreated (control group; Ctrl). (A) Cell adhesion ability was evaluated at $24 \mathrm{~h}$ after cell seeding. The level in the Ctrl group was set as $100 \%$. (B) Proliferation rate was compared. Levels at 1 day (1d) were set as 1 . (C) Keratocytes were treated with or without NSC 23766 for 3 days. Cell migration ability was measured by transferring cells to a transwell insert and analyzed after $24 \mathrm{~h}$. (D) Gene expression of MMPs after 3 days of treatment. Levels in the Ctrl group were set as $1 . *_{p}<0.05$. $* * p<0.001$. ns no significant difference $(\mathrm{p} \geq 0.05)$.

Rho-family of small GTPases regulates cytoskeletal remodeling, as molecular switches to transmit signals of mechanics, including stiffness. Racl and ROCK are central members of Rho-family. Inhibitors of Rac1 (NSC 23766) and ROCK (Y27632) were used in this study. It was found that NSC 23766 treatment decreased the expression of keratocyte phenotype markers (ALDH3A1, 63.67\%, $p<0.05$; CD34, 81.00\%, $p \geq$ 0.05 ; LUM, 70.67\%, $p<0.05)$ as compared to the control, untreated group (Figure 5B). However, similar results were not seen for Y-27632 treatment (Figure 5B). In addition, F-actin staining was performed to show the cytoskeletal reorganization after NSC 23766 treatment (Figure 5C). Cell area and the cellular aspect ratio were quantified (Figures 5D-5G). The cell area significantly decreased (29.79\%) after NSC 23766 treatment as compared to control (Figure 5D, $p<0.05$ ), while no difference was found for the cellular aspect ratio (Figure $5 \mathrm{~F}$, $p \geq 0.05$ ).

3.6. NSC 23766 Regulates Cell Adhesion, Proliferation, and Migration of Cultured Keratocytes. The effects of NSC 23766 treatment on cell adhesion, proliferation, and migration were evaluated (Figure 6). As compared to the control group, NSC 23766 treatment significantly increased the cell adhesion ratio $(128.37 \%, p<0.001)$ (Figure 6A), but inhibited the cell proliferation rate at day $4(3.22 \pm 0.20$ vs $4.52 \pm 0.31, p<0.001$, Figure $6 \mathrm{~B})$. As for cell migration ability, a $17.63 \%$ decrease $(p<0.05)$ was shown in the NSC 23766 treated group as compared to the control group (Figure 6C). Consistently, expression of four out of six studied MMPs was significantly downregulated ( $p<0.05$ for MMP1, MMP3, $M M P 9$, and MMP12, Figure 6D) in the NSC 23766-treated group as compared to the control group.

\section{DISCUSSION}

Substrate stiffness is one of the important microenvironmental factors that could regulate cell fate and the cell behavior. The effect of substrate stiffness has been studied in many different cell types, by culturing cells on soft and stiff materials. This includes mesenchymal stem cells, ${ }^{28}$ muscle stem cells, ${ }^{29}$ endothelial cells, ${ }^{30}$ deep infiltrating endometriotic stromal cells, ${ }^{31}$ among others. The main cell type in the corneal stroma is keratocytes, which are the cells responsible for the regulation of key factors that are important for the function of the cornea, and therefore these cells influence the vision. Although there are some previous reports on the effects of substrate stiffness on keratocyte cell culture, those studies mainly focus on the change of the cell morphology and the transformation of keratocytes. $5,12,13$ In contrast, our study focuses on the expression of keratocyte phenotype markers at the molecular level, which has not been described in previous studies and which is a valuable addition to current knowledge. In our study, the substrate with the equivalent of physiological corneal stiffness $(25 \mathrm{kPa})$ was found beneficial for in vitro keratocyte culture, as compared to TCP $\left(\sim 10^{6} \mathrm{kPa}\right)$, the widely used commercial plates for most cell types. To our knowledge, this is the first time that the importance of physiological corneal stiffness $(25 \mathrm{kPa})$ for keratocyte and corneal function is confirmed and directly supported by experimental data at the molecular level. This information contributes to the topic of material microenvironment and its importance in regulating cell fate, as well as to the development of corneal tissue engineering, because it is required that keratocytes preserve their phenotype and function within an artificial corneal model.

Previously, Dreier and collaborators cultured corneal fibroblasts on TCP and soft substrates $(4-71 \mathrm{kPa})$ within a fibrosis-inducing culture system, that is, TGF- $\beta 1$-supplemented 
medium. ${ }^{12}$ They found that the myofibroblast transformation was more obvious when the cells were cultured on TCP, as concluded by solely detecting the expression of $\alpha$-SMA. However, in our comparison of TCP and CCP, only a low expression of $\alpha$-SMA was found by cultured keratocytes. The reason for this could be attributed to the different culture systems used in these two studies. Serum is known to cause the phenotype transition from keratocyte to fibroblast. ${ }^{4}$ In Dreier et al.'s study, isolated human keratocytes were cultured in a $10 \%$ FBS-supplemented medium to obtain corneal fibroblasts. Their study focused on the transition from corneal fibroblast toward myofibroblast by culturing the fibroblasts on TCP and soft substrates. In our current study, isolated human keratocytes were cultured in a $2 \%$ FBS-supplemented medium. This culture system was set up in our group and has been welltested in our previous research work. ${ }^{32}$ In this culture condition, cells expressed the most important keratocyte markers, including ALDH, LUM, CD34, and KERA, but only a weak expression of $\alpha$-SMA. ${ }^{32}$ Therefore, this lower concentration of FBS decreased the phenotype transition effect of serum and preserved the keratocyte phenotype. Based on that, our study evaluated the effect of substrate stiffness on the phenotype markers expression of keratocytes and cell behaviors.

The main markers of the keratocyte phenotype include ALDH, CD34, LUM, and KERA. ALDH is a water-soluble protein that possess enzymatic activity. High concentration of $\mathrm{ALDH}$ in corneal tissue makes the refractive index of keratocytes match with their ECM and therefore contributes to corneal transparency. ${ }^{33}$ Correspondingly, loss of ALDH can result in corneal haziness. ${ }^{34}$ Therefore, high expression of $\mathrm{ALDH}$ in keratocytes is important for achieving transparency in tissue-engineered cornea. ALDH3A1 and ALDH1A1 are the prominent isozymes in human cornea. Decrease of ALDH3A1 has been found during the transformation of keratocytes to myofibroblasts. ${ }^{35}$ In our study, keratocytes cultured on plates with physiological corneal stiffness (25 kPa group) expressed much higher $A L D H 3 A 1$ and $A L D H 1 A 1$, as compared to cells cultured on TCP (Figure 2A). Physiological vitreous body stiffness (1 kPa group) also promoted the expression of these two genes, but to a lesser extent than in the $25 \mathrm{kPa}$ group (Figure 2A). These results collectively show that keratocytes prefer to be cultured in a condition more equivalent to physiological corneal stiffness in order to maintain their expression of ALDH3A1 and ALDH1A1.

CD34 is a cell surface glycoprotein. It is a hematopoietic stem cell marker and has been used as a typical phenotype marker of keratocytes as well. ${ }^{4}$ LUM and KERA are both major proteoglycans that are present in the corneal stroma. LUM is important for corneal transparency by regulating collagen fibril growth and spacing. ${ }^{4}$ KERA regulates collagen fibril diameter and organization. ${ }^{36}$ An LUM-null mouse has a profound phenotype alternation; a phenomenon that is not seen in a KERA-null mouse. ${ }^{37}$ This could be explained by the fact that the expression of KERA is regulated by LUM. ${ }^{37}$ With that in mind and the fact that no significant difference was observed in KERA between TCP and CCP (Figure 1A,B), KERA was not subsequently evaluated in our study. Both CD34 and LUM showed similar expression profiles as ALDH, when keratocytes were cultured on plates with different stiffnesses (Figure 2A). In summary, these results indicate the importance of physiological corneal stiffness for keratocyte and corneal function, which is expected by researchers, but to our knowledge has never previously been directly confirmed by experimental data, until now in the present study. However, the optimal range of stiffness for in vitro keratocyte culture still needs to be determined in future studies.

We furthermore studied the effect of substrate stiffness on cell behaviors of keratocytes including cell adhesion, proliferation, and migration. Obvious differences were again found between groups. Studies were further performed on the inherent mechanisms of the cells, through which substrate stiffness regulates the cell fate of keratocytes. The cytoskeletal structure is important for the phenotype and behaviors of cultured cells. ${ }^{14,15}$ In our study, keratocytes cultured on $25 \mathrm{kPa}$ plates showed larger cell area as compared to those cultured on TCP (Figure 4A-C), indicating a role of the cytoskeletal structure in transmitting the physical signal of the substrate stiffness. Treatment by Cytochalasin D (Cyto D), which disrupts actin filaments, ${ }^{27}$ modified the cell shape into a round appearance, and the expression of phenotype markers decreased to a very low level (Figure 5A,B). Inhibition of Rac1 by NSC 23766 showed similar effects as Cyto D treatment on the expression of keratocyte phenotype markers. NSC 23766 treatment also resulted in altered cytoskeletal structure (cell area), cell adhesion, proliferation and migration (Figures 5 and 6). These results collectively suggest that substrate stiffness regulates the phenotype marker expression and cell behavior of keratocyte through Rac1-mediated cytoskeletal reorganization.

Racl could be activated when combined with guanosine triphosphate (GTP) and inactivated when combined with guanosine diphosphate (GDP). Therefore, Rac1 can work as a molecular switch, regulating the cytoskeletal structure and subsequently influencing multiple cell functions. ${ }^{38}$ Previous studies ${ }^{16,39}$ and our current study (Figures 5 and 6) demonstrate the importance of Racl in transmitting the physical signal to intracellular signaling. Racl inhibition by NSC 23766 mimicked the phenotype shift of keratocytes that was seen when the cells were cultured on TCP instead of soft plates. Culturing keratocytes on plates with physiological corneal stiffness was shown to be good for the promotion of the keratocyte phenotype marker expression and therefore making in vitro cell study more realistic and valuable, as compared to culturing on TCP. However, in economic terms, current commercial soft plates are expensive as routine consumables for a majority of labs, which hampers their popularization. Based on the information shown in this study, it is suggested that activating Racl in keratocytes cultured on TCP plates, might result in similar cell phenotype and behavior as in keratocytes cultured on soft plates. Establishing a method of specifically activating Rac1 would be of great significance for scientific research in this field.

It has been reported that corneal tissue tends to get stiffer with age because of alternations in the microstructure of the stromal tissue, such as increase of stromal fibril diameter ${ }^{40}$ and interfibrillar cross-linking. ${ }^{41}$ This study and previous studies suggest that a stiff matrix could induce phenotype drift and myofibroblast transformation, and thereby destroy the original normal functions of the cells. ${ }^{12,42}$ Furthermore, stiffened corneal tissue is one possible reason for elevated intraocular pressure, ${ }^{42}$ which is the main risk factor of glaucoma. ${ }^{43}$ It is of great significance to reveal the systematic effect of stiffness on corneal cells and elucidate its inherent mechanism. Potential treatments in clinics may be developed in the future by regulating the cytoskeletal structure and Racl activation, which 
could reverse the phenotype drift of keratocytes, and consequent deteriorated corneal tissue functions, caused by age-related tissue stiffening.

\section{CONCLUSIONS}

In summary, the current study evaluated the effects and mechanisms of substrate stiffness on keratocyte phenotype marker expression and different cell behaviors, including cell adhesion, proliferation, and migration. Physiological corneal stiffness $(25 \mathrm{kPa})$ was found to be beneficial for preservation of the phenotype of cultured keratocytes. Cytoskeletal structures were different in keratocytes when they were cultured on substrates with different stiffness, which affects the regulation of the cell phenotype marker expression and cell behavior by Rac1 involvement. The results of this study emphasize the importance of material stiffness in keratocyte culture in vitro and will potentially contribute to the further improvement of corneal tissue engineering.

\section{ASSOCIATED CONTENT}

\section{(s) Supporting Information}

The Supporting Information is available free of charge at https://pubs.acs.org/doi/10.1021/acsbiomaterials.0c00510.

Antibody Information; probe information; primer information; effects of COL I and COL V on the Expression of keratocyte Phenotype Markers; and supporting Methods (PDF)

\section{AUTHOR INFORMATION}

\section{Corresponding Authors}

Jialin Chen - Department of Pathogenic Biology and Immunology, School of Medicine and Jiangsu Key Laboratory for Biomaterials and Devices, Southeast University, Nanjing 210009, China; Department of Integrative Medical Biology, Anatomy, Umeå University, Umeå SE-901 87, Sweden; (1) orcid.org/0000-0001-5038-3474; Phone: +86-2583272500; Email: jialin.chen@seu.edu.cn; Fax: +86-2583324887

Patrik Danielson - Department of Integrative Medical Biology, Anatomy and Department of Clinical Sciences, Ophthalmology, Umeå University, Umeå SE-901 87, Sweden; Phone: +46 (0) 9078658 93; Email: patrik.danielson@umu.se

\section{Authors}

Ludvig J. Backman - Department of Integrative Medical Biology, Anatomy and Department of Community Medicine and Rehabilitation, Physiotherapy, Umeå University, Umeå SE-901 87, Sweden

Wei Zhang - Department of Integrative Medical Biology, Anatomy, Umeå University, Umeå SE-901 87, Sweden; Jiangsu Key Laboratory for Biomaterials and Devices and Department of Physiology, School of Medicine, Southeast University, Nanjing 210009, China; 이이. orcid.org/0000-0003-2700-6739

Chen Ling - Department of Orthopaedic Surgery, Institute of Digital Medicine, Nanjing First Hospital, Nanjing Medical University, Nanjing 210000, China

Complete contact information is available at: https://pubs.acs.org/10.1021/acsbiomaterials.0c00510

\section{Notes}

The authors declare no competing financial interest.

\section{ACKNOWLEDGMENTS}

The authors thank Dr. Marta Słoniecka, Dr Xin Zhou, and Roine El-Habta for scientific advice. This work was financially supported by the national Swedish Research Council (grant 2017-01138), the Swedish Society of Medicine (grant SLS504541), the Cronqvist foundation (grant SLS-691711), the foundation Kronprinsessan Margaretas Arbetsnämnd för synskadade (KMA, grant 2013/10), the foundation Ögonfonden, and via federal funds through a regional agreement (ALF) between Umeå University and the Västerbotten County Council (VLL-549761). This work was also supported by the National Natural Science Foundation of China (31900962, 81901903), the Natural Science Foundation of Jiangsu Province (BK20190354, BK20190356), the Fundamental Research Funds for the Central Universities, the Funds for Zhishan Young Scholars (Southeast University), and the Scientific Research Foundation for Returned Scholars (1124007113).

\section{REFERENCES}

(1) Tan, D. T.; Dart, J. K.; Holland, E. J.; Kinoshita, S. Corneal transplantation. Lancet 2012, 379, 1749-1761.

(2) Zhang, M. C. [Strategies to resolve the problem of lack of keratoplasty materials in China]. Zhonghua Yanke Zazhi 2007, 43, 289-292.

(3) Guan, H. J. [Present status and development of prevention of blindness and ophthalmic epidemiologic studies in China]. Zhonghua Yanke Zazhi 2010, 46, 938-943.

(4) Kumar, P.; Pandit, A.; Zeugolis, D. I. Progress in Corneal Stromal Repair: From Tissue Grafts and Biomaterials to Modular Supramolecular Tissue-Like Assemblies. Adv. Mater. 2016, 28, 53815399.

(5) Petroll, W.; Lakshman, N. Fibroblastic Transformation of Corneal Keratocytes by Rac Inhibition is Modulated by Extracellular Matrix Structure and Stiffness. J. Funct. Biomater. 2015, 6, 222-240.

(6) Ma, Y.; Lin, M.; Huang, G.; Li, Y.; Wang, S.; Bai, G.; Lu, T. J.; $\mathrm{Xu}$, F. 3D Spatiotemporal Mechanical Microenvironment: A Hydrogel-Based Platform for Guiding Stem Cell Fate. Adv. Mater. 2018, 30, No. e1705911.

(7) Lim, B.; Woodward, W. A.; Wang, X.; Reuben, J. M.; Ueno, N. $\mathrm{T}$. Inflammatory breast cancer biology: the tumour microenvironment is key. Nat. Rev. Cancer 2018, 18, 485-499.

(8) Zhang, W.; Chen, J.; Backman, L. J.; Malm, A. D.; Danielson, P. Surface Topography and Mechanical Strain Promote Keratocyte Phenotype and Extracellular Matrix Formation in a Biomimetic 3D Corneal Model. Adv. Healthc. Mater. 2017, 6, 1601238.

(9) Molladavoodi, S.; Kwon, H.-J.; Medley, J.; Gorbet, M. Human corneal epithelial cell response to substrate stiffness. Acta Biomater. 2015, 11, 324-332.

(10) Moers, K.; Steinberg, T.; Schlunck, G.; Reinhard, T.; Tomakidi, P.; Eberwein, P. Substrate elasticity as biomechanical modulator of tissue homeostatic parameters in corneal keratinocytes. Exp. Cell Res. 2013, 319, 1889-1901.

(11) Last, J. A.; Thomasy, S. M.; Croasdale, C. R.; Russell, P.; Murphy, C. J. Compliance profile of the human cornea as measured by atomic force microscopy. Micron 2012, 43, 1293-1298.

(12) Dreier, B.; Thomasy, S. M.; Mendonsa, R.; Raghunathan, V. K.; Russell, P.; Murphy, C. J. Substratum compliance modulates corneal fibroblast to myofibroblast transformation. Invest. Ophthalmol. Visual Sci. 2013, 54, 5901-5907.

(13) Lakshman, N.; Petroll, W. M. Growth factor regulation of corneal keratocyte mechanical phenotypes in 3-D collagen matrices. Invest. Ophthalmol. Visual Sci. 2012, 53, 1077-1086.

(14) Gladilin, E.; Ohse, S.; Boerries, M.; Busch, H.; Xu, C.; Schneider, M.; Meister, M.; Eils, R. TGFbeta-induced cytoskeletal remodeling mediates elevation of cell stiffness and invasiveness in NSCLC. Sci. Rep. 2019, 9, 7667. 
(15) Yao, Y.; Gu, X.; Liu, H.; Wu, G.; Yuan, D.; Yang, X.; Song, Y. Metadherin regulates proliferation and metastasis via actin cytoskeletal remodelling in non-small cell lung cancer. Br. J. Cancer 2014, $111,355-364$.

(16) Pasapera, A. M.; Plotnikov, S. V.; Fischer, R. S.; Case, L. B.; Egelhoff, T. T.; Waterman, C. M. Rac1-dependent phosphorylation and focal adhesion recruitment of myosin IIA regulates migration and mechanosensing. Curr. Biol. 2015, 25, 175-186.

(17) Xu, B.; Song, G.; Ju, Y.; Li, X.; Song, Y.; Watanabe, S. RhoA/ ROCK, cytoskeletal dynamics, and focal adhesion kinase are required for mechanical stretch-induced tenogenic differentiation of human mesenchymal stem cells. J. Cell. Physiol. 2012, 227, 2722-2729.

(18) Petroll, W. M.; Miron-Mendoza, M. Mechanical interactions and crosstalk between corneal keratocytes and the extracellular matrix. Exp. Eye Res. 2015, 133, 49-57.

(19) Chou, S.-F.; Lai, J.-Y.; Cho, C.-H.; Lee, C.-H. Relationships between surface roughness/stiffness of chitosan coatings and fabrication of corneal keratocyte spheroids: Effect of degree of deacetylation. Colloids Surf., B 2016, 142, 105-113.

(20) Sun, C.-C.; Chou, S.-F.; Lai, J.-Y.; Cho, C.-H.; Lee, C.-H. Dependence of corneal keratocyte adhesion, spreading, and integrin beta 1 expression on deacetylated chitosan coating. Mater. Sci. Eng., C 2016, 63, 222-230.

(21) Chen, J.; Chen, P.; Backman, L. J.; Zhou, Q.; Danielson, P. Ciliary Neurotrophic Factor Promotes the Migration of Corneal Epithelial Stem/progenitor Cells by Up-regulation of MMPs through the Phosphorylation of Akt. Sci. Rep. 2016, 6, 25870.

(22) Chen, J.; Lan, J.; Liu, D.; Backman, L. J.; Zhang, W.; Zhou, Q.; Danielson, P. Ascorbic Acid Promotes the Stemness of Corneal Epithelial Stem/Progenitor Cells and Accelerates Epithelial Wound Healing in the Cornea. Stem Cells Transl. Med. 2017, 6, 1356-1365. (23) Chen, J.; Zhang, W.; Kelk, P.; Backman, L. J.; Danielson, P. Substance $\mathrm{P}$ and patterned silk biomaterial stimulate periodontal ligament stem cells to form corneal stroma in a bioengineered threedimensional model. Stem Cell Res. Ther. 2017, 8, 260.

(24) Boettiger, D. Mechanical control of integrin-mediated adhesion and signaling. Curr. Opin. Cell Biol. 2012, 24, 592-599.

(25) Chen, P.; Parks, W. C. Role of matrix metalloproteinases in epithelial migration. J. Cell. Biochem. 2009, 108, 1233-1243.

(26) Discher, D. E.; Janmey, P.; Wang, Y. L. Tissue cells feel and respond to the stiffness of their substrate. Science 2005, 310, 11391143

(27) Yin, Z.; Chen, X.; Song, H.-x.; Hu, J.-j.; Tang, Q.-m.; Zhu, T.; Shen, W.-1.; Chen, J.-1.; Liu, H.; Heng, B. C.; Ouyang, H.-W. Electrospun scaffolds for multiple tissues regeneration in vivo through topography dependent induction of lineage specific differentiation. Biomaterials 2015, 44, 173-185.

(28) Li, C. X.; Talele, N. P.; Boo, S.; Koehler, A.; Knee-Walden, E.; Balestrini, J. L.; Speight, P.; Kapus, A.; Hinz, B. MicroRNA-21 preserves the fibrotic mechanical memory of mesenchymal stem cells. Nat. Mater. 2017, 16, 379-389.

(29) Gilbert, P. M.; Havenstrite, K. L.; Magnusson, K. E. G.; Sacco, A.; Leonardi, N. A.; Kraft, P.; Nguyen, N. K.; Thrun, S.; Lutolf, M. P.; Blau, H. M. Substrate elasticity regulates skeletal muscle stem cell selfrenewal in culture. Science 2010, 329, 1078-1081.

(30) Wood, J. A.; Shah, N. M.; McKee, C. T.; Hughbanks, M. L.; Liliensiek, S. J.; Russell, P.; Murphy, C. J. The role of substratum compliance of hydrogels on vascular endothelial cell behavior. Biomaterials 2011, 32, 5056-5064.

(31) Matsuzaki, S.; Canis, M.; Pouly, J.-L.; Darcha, C. Soft matrices inhibit cell proliferation and inactivate the fibrotic phenotype of deep endometriotic stromal cells in vitro. Hum. Reprod. 2016, 31, 541-553.

(32) Słoniecka, M.; Le Roux, S.; Boman, P.; Byström, B.; Zhou, Q.; Danielson, P. Expression Profiles of Neuropeptides, Neurotransmitters, and Their Receptors in Human Keratocytes In Vitro and In Situ. PLoS One 2015, 10, No. e134157.

(33) Jester, J. V.; Moller-Pedersen, T.; Huang, J.; Sax, C. M.; Kays, W. T.; Cavangh, H. D.; Petroll, W. M.; Piatigorsky, J. The cellular basis of corneal transparency: evidence for 'corneal crystallins. J. Cell Sci. 1999, 112, 613-622.

(34) Jester, J. V.; Petroll, W. M.; Cavanagh, H. D. Corneal stromal wound healing in refractive surgery: the role of myofibroblasts. Stem Cell Res. Ther. 1999, 18, 311-356.

(35) Pei, Y.; Reins, R. Y.; McDermott, A. M. Aldehyde dehydrogenase $(\mathrm{ALDH}) 3 \mathrm{Al}$ expression by the human keratocyte and its repair phenotypes. Exp. Eye Res. 2006, 83, 1063-1073.

(36) Liu, C.-Y.; Birk, D. E.; Hassell, J. R.; Kane, B.; Kao, W. W.-Y. Keratocan-deficient mice display alterations in corneal structure. $J$. Biol. Chem. 2003, 278, 21672-21677.

(37) Carlson, E. C.; Liu, C.-Y.; Chikama, T.-i.; Hayashi, Y.; Kao, C. W.-C.; Birk, D. E.; Funderburgh, J. L.; Jester, J. V.; Kao, W. W.-Y. Keratocan, a cornea-specific keratan sulfate proteoglycan, is regulated by lumican. J. Biol. Chem. 2005, 280, 25541-25547.

(38) Ridley, A. J. Life at the leading edge. Cell 2011, 145, 10121022.

(39) Chaudhuri, O.; Koshy, S. T.; Branco da Cunha, C.; Shin, J.-W.; Verbeke, C. S.; Allison, K. H.; Mooney, D. J. Extracellular matrix stiffness and composition jointly regulate the induction of malignant phenotypes in mammary epithelium. Nat. Mater. 2014, 13, 970-978.

(40) Daxer, A.; Misof, K.; Grabner, B.; Ettl, A.; Fratzl, P. Collagen fibrils in the human corneal stroma: structure and aging. Invest. Ophthalmol. Visual Sci. 1998, 39, 644-648.

(41) Malik, N.; Moss, S.; Ahmed, N.; Furth, A.; Wall, R.; Meek, K. Ageing of the human corneal stroma: structural and biochemical changes. Biochim. Biophys. Acta 1992, 1138, 222-228.

(42) Liu, B.; McNally, S.; Kilpatrick, J. I.; Jarvis, S. P.; O’Brien, C. J. Aging and ocular tissue stiffness in glaucoma. Surv. Ophthalmol. 2018, $63,56-74$.

(43) Leske, M. C.; Heijl, A.; Hussein, M.; Bengtsson, B.; Hyman, L.; Komaroff, E. Factors for glaucoma progression and the effect of treatment: the early manifest glaucoma trial. Arch. Ophthalmol. 2003, $121,48-56$. 